\title{
Computer Aided Medical Diagnosis for the Treatment of Sexually Transmitted Disease (Gonorrhea)
}

Adamu M. Ibrahim*, Fatima Isiaka ${ }^{1}$

*School of Computing, University of Leeds, UK

${ }^{1}$ Sheffield Hallam University, Department of Computing, UK

\begin{tabular}{l} 
A R T I C L E I N F O \\
\hline Article history: \\
Received: 19 January, 2017 \\
Accepted: 06 February 2017 \\
Online: 25 February, 2017 \\
\hline Keywords: \\
Structured systems analysis \\
Treatment \\
Sexually transmitted diseases \\
Expert systems
\end{tabular}

\section{Introduction}

Recently, assessment of the WHO study on the condition of clinical facilities in developing countries suggest that there is considerable efficiency in the provision of medical services particularly to the rural dwellers where the services are available, they are very expensive and unaffordable to the average citizen. This has resulted in prolonged suffering and death. Moreover, the slow process of diagnosis trail and error of disease can be fatal when a patient is at terminal point of his disease. The diagnosis and treatment of Sexually Transmitted Diseases is what the researcher deemed very important, since the practice of prostitution both at local and international level and widespread promiscuity among our teenagers, youths and even married ones in on geometric increase. The sexually transmitted disease (STD) is a group of diseases transmitted during sexual intercourse or close sexual contact with an infected person such Sexually Transmitted Disease includes Syphilis, Gonorrhoea, Chancroed, Non Gonococal Urethritis in the male, Trichomonal Vaginities in the female, Herpes Genitalis, Condylomata Accuminata, Molluscum Contagiosum and lately the Acquired Immune Deficiency Syndrome (AIDS) [6]. In this project, the researcher's interest will only be based on the called Gonorrhoea.

\footnotetext{
*Adamu Ibrahim, University of Leeds, imailafiya@yahoo.com
}

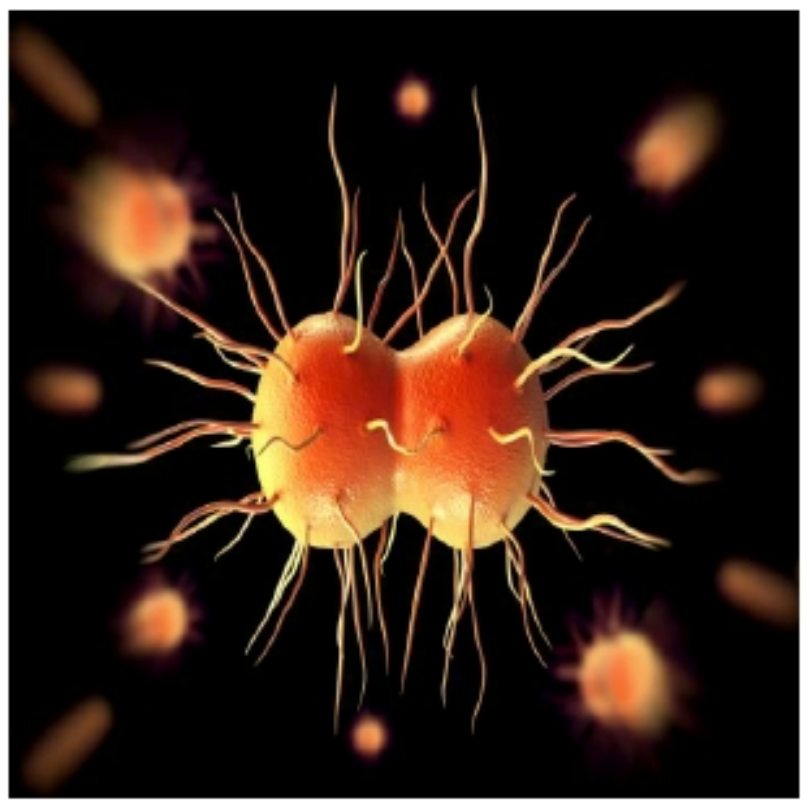

Fig. 1: The gonorrhoea bacteria: Courtesy Google Images 


\subsection{Gonorrhea}

This is a sexually transmitted infection due to Neisseria Gonorrhoea (the gonococcus) (Figure 1), which effects primarily the anterior urethra in the male and the urethra and cervix in female. In recent years there has been a great increase in the cases of gonorrhoea throughout the world; approximately 60,000 cases are seen annually in the clinics of England and this figure is even higher in the case of Nigeria and other African countries.

After three to five days incubation period in male, the following symptoms and signs will be observed; Dysuria, Urethral discharge (i.e Seropurulent becoming purulent and yellowish) Reddening of urethral meatus etc. Gonococcal protitis common in male homosexuals and is often symptomless.

In female, Gonorrhoea is frequently a symptomatic and maynever be diagnosed on clinical grounds. Almost three quarters of all female patients attend initially as contacts of male cases and not because of their signs or symptoms. The signs and symptomsin female includes symslight dysuris, slight or moderate vaginal discharge, which is often unnoticed by patient, reddening or acute erosion of the cervix, mucopurulent cervical plug, copious vaginal discharge is more likely to due to concomitant trichomonal vaginities and lower abdominal pain and tenderness is also present, with thickening and tenderness of the affected tube on bimanual palpation. Infertility following bilateral gonococcal salpingitis is now uncommon.

To identify the problem, we look at current trend in information technology. Though the world's information technology is rapidly changing, thanks to the likes of Bill Gates and his cohorts of Microsoft Corporation and International Electronic (INTEL) of U.S.A. In developing countries like Nigeria, the case is contrary, we see a different trend in science and its development altogether. While some private and government hospitals are now computerized most especially in areas like X-Ray services, Laboratory services and Ultrasound services, the STD units, which have not been much computerized, need an urgent attention. Some of the problems adherent to the manual operation of the STD unit includes:

- Inaccurate diagnosis of the STDs, which may lead to wrong drug prescription, and at the end may lead to ultimate destruction of the organ involved, especially in women

- Bulkiness of the records because of the rate at which both the teenagers, youths and even the married go about patronising prostitutes, engaging in unprotected sex and thereby, spreading the STDs.

- Monotonous and repetitive computation.

- Retirement, transfer or even death of human expert, which when happen goes with his/her knowledge.

Viewing the above-enumerated problems, Computer-Aided Medical Diagnosis for the Treatment of gonorrhoea or STD, as the title of this project study, becomes very imperative.

\subsection{Objective of the paper}

Most a times, when the human expert is not available, the diagnosis and treatment of the Sexually Transmitted Disease (STD) seems to be very difficult to the infected ones. Therefore, the objective of this study is to bring together the combine knowledge of different human expertise and use it to design and implement an automated system that can aid the diagnosis of the above mentioned sexually transmitted disease and gives adequate drug prescriptions. The paper may also cover some administrative aspect for the STD patients.

\section{Review of some Related Work}

This paper also make reference to the works held in the field of computer application to Medicare. Recalled that $[9][12][7][8][5][4][13][14]$, stated that "Everything must have a beginning, and that must be linked to something that went before". From the above statement, one can see that concepts are formed from the reflections of what one reads, observed, examined and discussed with others. On this note, this paper reviewed some works and studies of different writers.

Blaya [3] in his book "E-health technologies show promise in developing countries" showed how health care is noted as priority for national development strategies. Therefore, government should not depict nonchalant attitudes towards reimbursing health sector. The fact that "the largest contributor to improved health is increase wealth and that poor health is the characteristic of poverty nation", [2]. Even the call for helping hands from paramedical staff to engage themselves in most doctor's responsibilities have failed because the situation had gone beyond the resident level.

Also, the various health care system that is concerned with regulating access to financing and organising the delivery of health care to the population, [10] has failed in developing countries with criticisms arising from the field of the method of implementation adopted. It was also stated that most developing countries system, employ a radical approach which cannot go beyond the paper scope [11] but rather prevented by such problems like poverty, environmental factors, individual life styles and the lack of manpower resources. Therefore, shortage of medical expertise can be viewed as one of the most major problems affecting health care delivery services.

Very often, people in developing countries who are critically ill are rushed overseas for special treatment because it is felt that medical facilities in under developed countries are inadequate [1]. This is partly because Computer-Aided Medical Diagnosis has become a reality in many developed countries. The need to adopt an Electronic Preventive Health Care Service Approach (EPHCSA), in the field of diagnosis, treatment and record system become imperatives, to help ease the workload of a medical officer. This electronic system will help a lot in curbing the problems of STD and other dreaded health care problems.

\section{Methodology}

Presently in developing countries like Nigeria, human expert does the diagnosis for the treatment of STD patients manually.

This starts when a patient comes to the hospital, he either registers if he/she has not registered before or presents his/her card bearing 


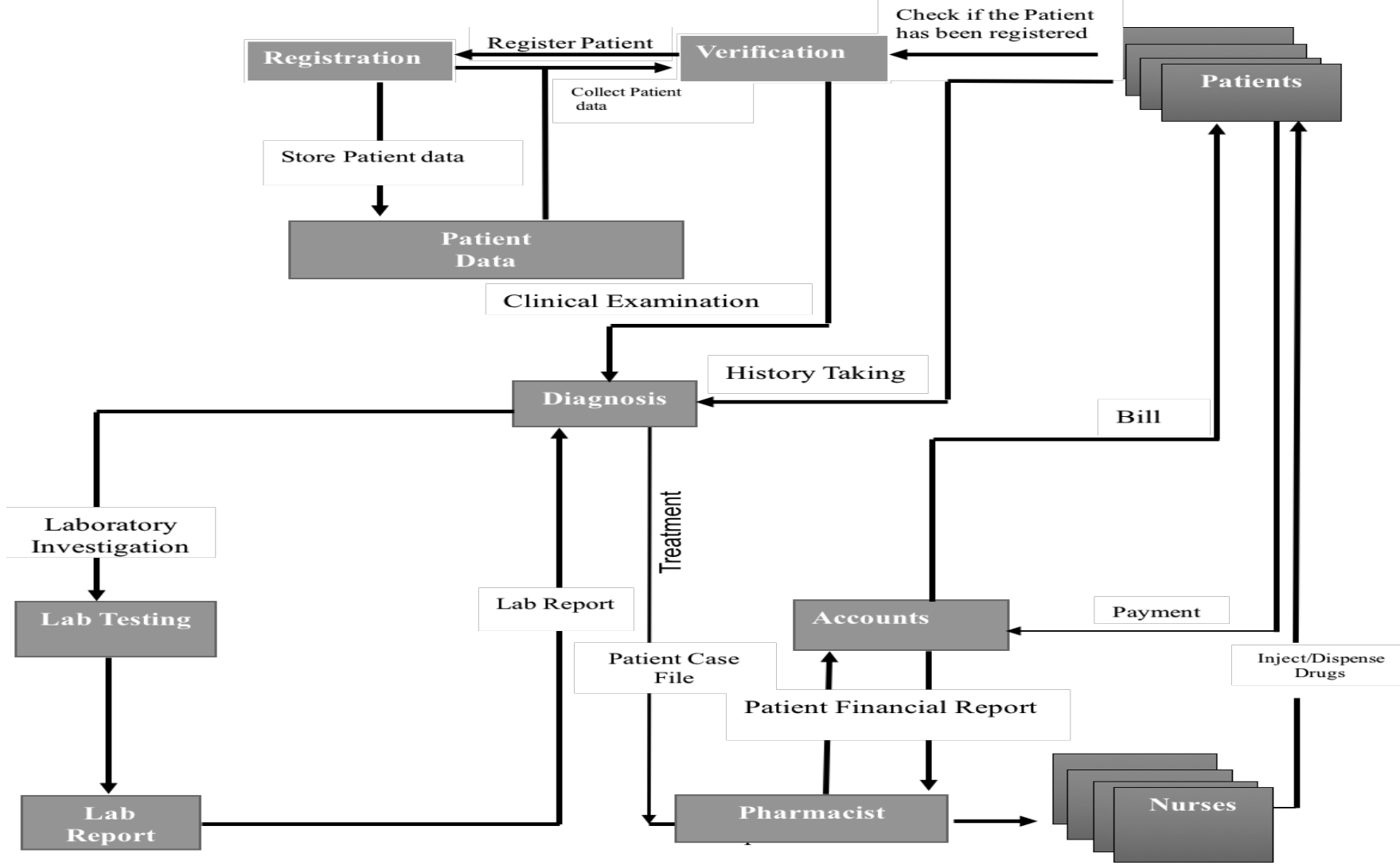

Fig. 2: Data Flow Diagram of Existing System

his/her case file. He/she then goes into doctors consulting room for some clinical examination. The doctor will now ascertain certain information from the patient about his/her illness then the patients is being diagnosed of an illness by the doctor in charge. In some cases, the doctor sends the patient for lab test if the patient has some conflicting issues. When this happens, the lab technologist will carry out the test and send the result back to the doctor, who will then prescribe drugs for the patient taking consideration on whether the patient is allergic to a certain drug or not. This process is indicated in the data flow diagram in Figure 2. The method adopted for the proposed system uses the SSADM, which is widely used in developed countries especially in the UK, a requirement for computing projects. The method sets out a series of techniques and processes, and guidelines for recording and communicating information concerning system development project both in word-based and diagrammatic form as shown in Figure 3.

Control from diagnosis and treatment shows initialization of counter, option of presence of symptoms is logical. For every selection, the counter increases to a limit, for example number of symptoms that result to gonorrhea is six which the system identifies before giving suggestions for treatment and drugs.

These results are then save and printed out. In cases where the number of symptoms selected is not enough to term the ailment as gonorrhea, the patient is sent for lab test and to see a medical practitioner.

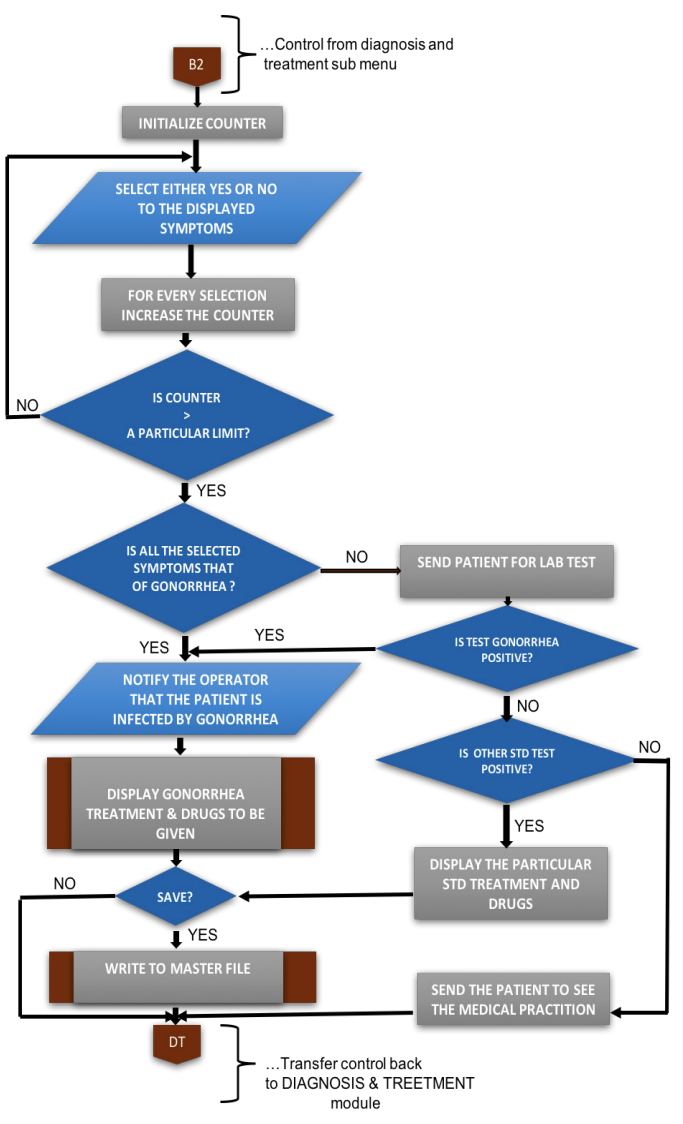

Fig. 3: Data Flow Diagram of Proposed System 


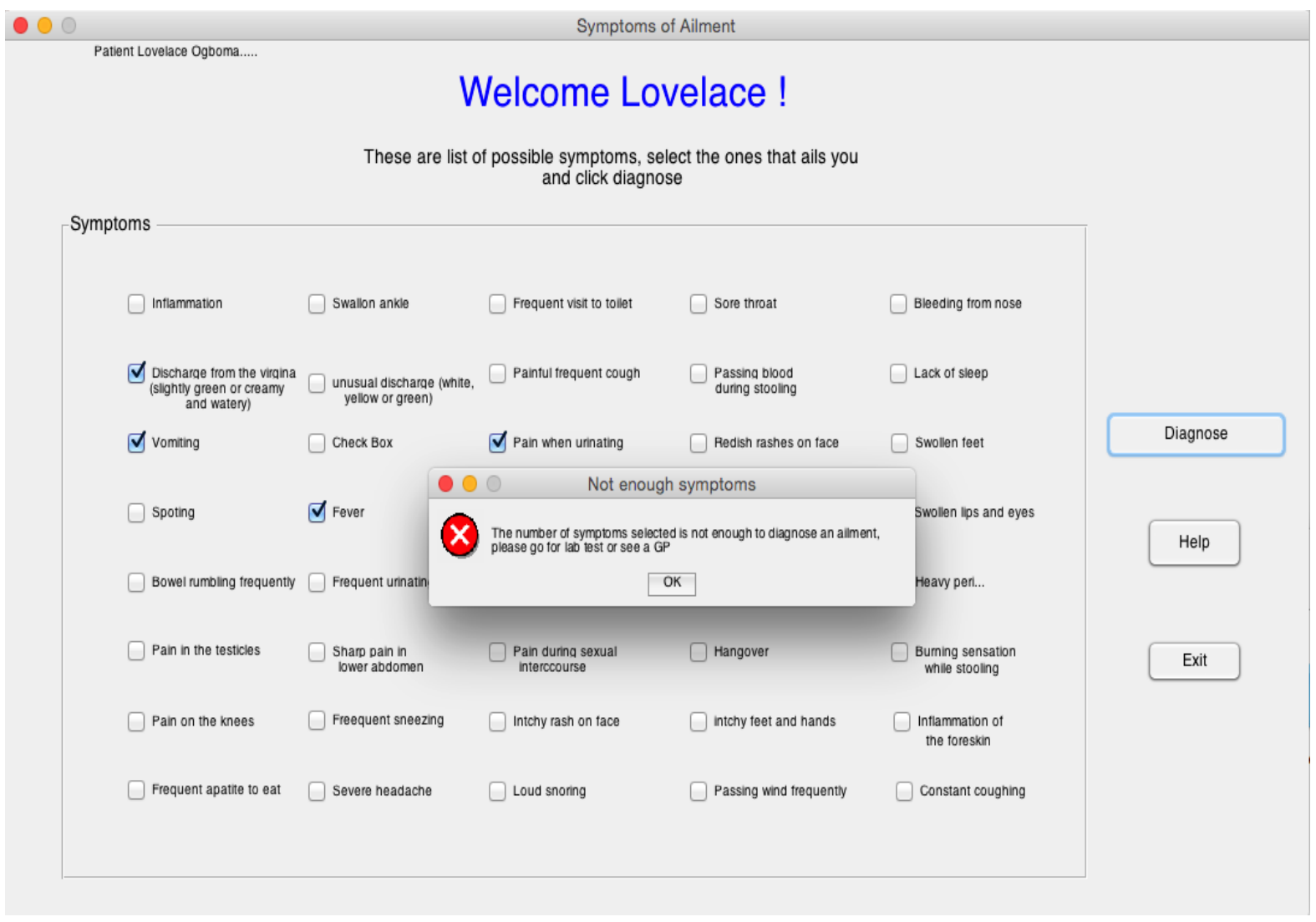

Fig. 4: Interphase with list of Symptoms for diagnosing different ailment

The patient case file will then be given to the pharmacist who will now select the drugs and give the information to the account section on how much is the cost of drugs. The account section prepares the bill for the patient, when the patient pays, the section will give a feedback to the pharmacist, who will then dispense the drugs to the patient through the nurse. In summary, the problems with existing system of diagnosis for the treatment of gonorrhea

includes time wastage, inaccurate diagnosis sometimes poor patient case file management/retrieval and absence of human expert permanence. The merits of the proposed system include:

- reduction in time contact between patient and doctor

- permanent data source

- accurate diagnosis

- accurate patent data management

- reduction in doctor's workload

- fast diagnosis processing

- no side distraction

The primary of the proposed system is to achieve a new system which is more reliable than the old ones in terms of efficiency and service. With the introduction of a Computer Aided System the transaction is online, this allows for information to be obtained in time for maximum use. This will help the user to collect personal data of the patient, symptoms and subsequently display the drug prescription. The design took extensive use of figthe menu-driven approach which routes program of interest and ensures that the user inputs consistent access path information. The design provides necessary manual and automated control that help to maintain the data integrity and security.

\section{Analysis and Implementation}

To implement the task in the proposed system, Matlab was used for its capabilities in easily integrating program modules and, possesses simbiology control tools for diagnosing biological courses. As discussed at the beginning of this paper, gonorrhoea has

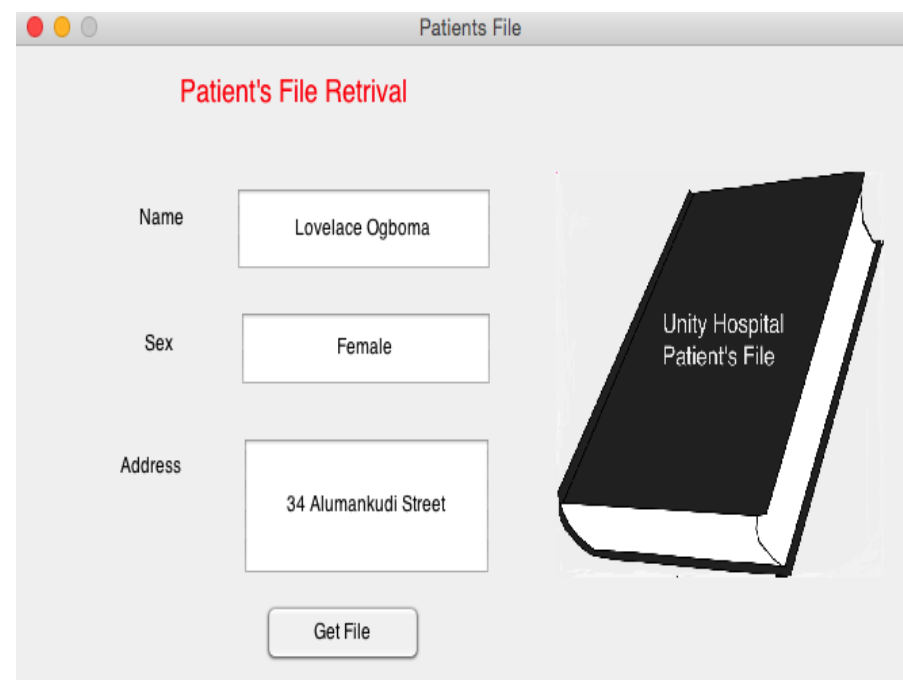

Fig. 5: Patients File Retrival 


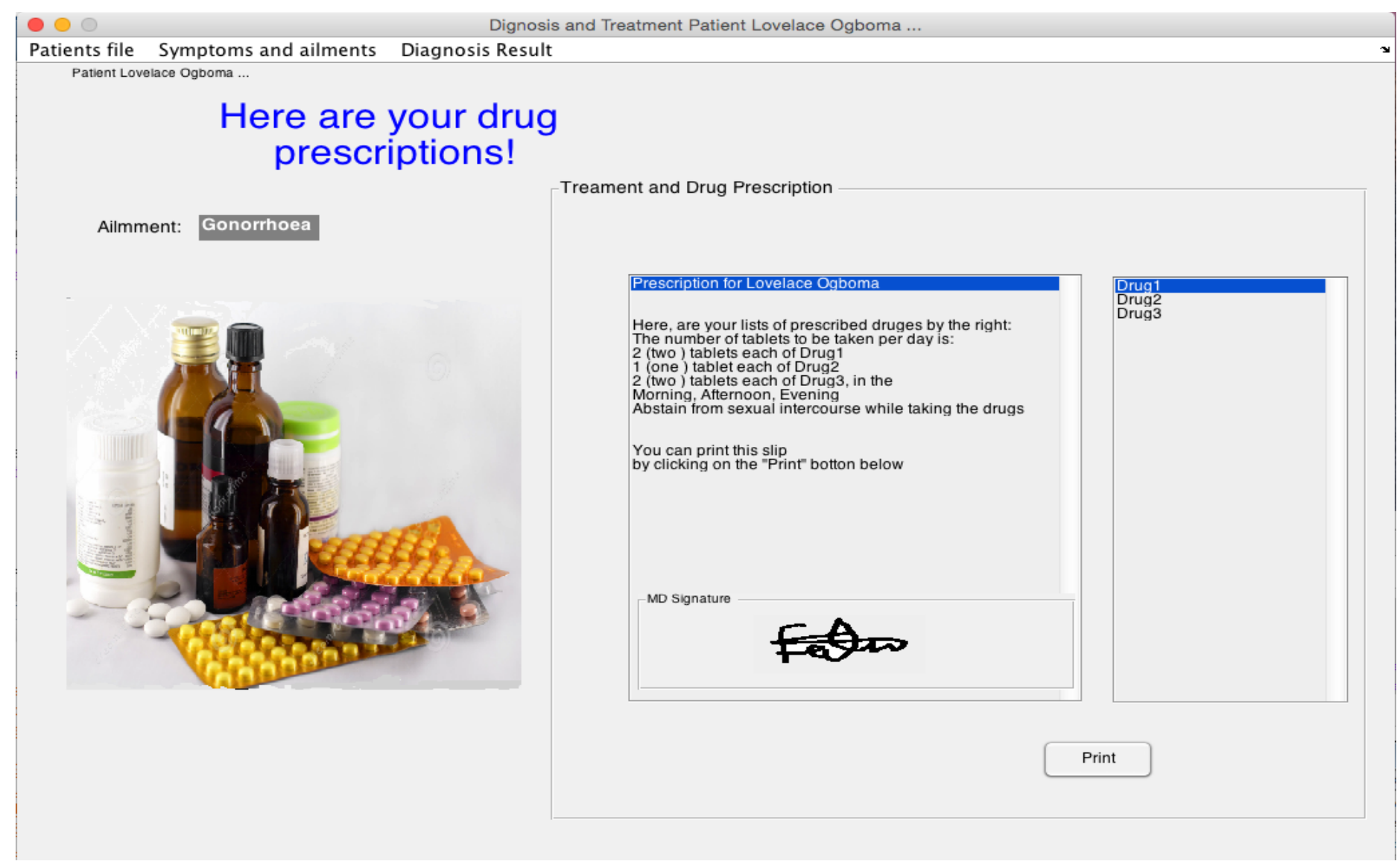

Fig. 6: Interphase displaying prescribed drugs and treatment

different symptoms for both male and female. For female, the patient could have fever, pain or a burning sensation while urinating, heavier period, discharge from the virgina that could be either creamy or slightly green in colour and watery, the frequent need to urinate, sore throat, sharp pain and the lower abdomen and finally pain upon engaging in sexual intercourse. While for male includes swelling of the foreskin, an unusual discharge from the tip of the penis, which may be the same colour has that of the female, pain or maybe tenderness in the testicles.

These number of symptoms from both parties must be entered and identified by the system (Figure 4) before a proper diagnosis can be made or else the patient may need to go for a lab test and see a practitioner. The system searches through all entries of record and selects the ones that matches the correct symptoms that is diagnosed for or recognises the ailment "gonorrhea", this is done through a random search and match algorithm with the Eq (1). This a simple optimization technique based on pure random search of text content. where $i=1, \ldots, n$ and limit of symptoms is equal to $\mathrm{n}$. This produces the convex surface containing all possible symptoms and their corresponding ailment. The range of variables or attributes in $y(i) \sum[-m, m] m$ is the number of symptoms required to identify an ailment in the database.

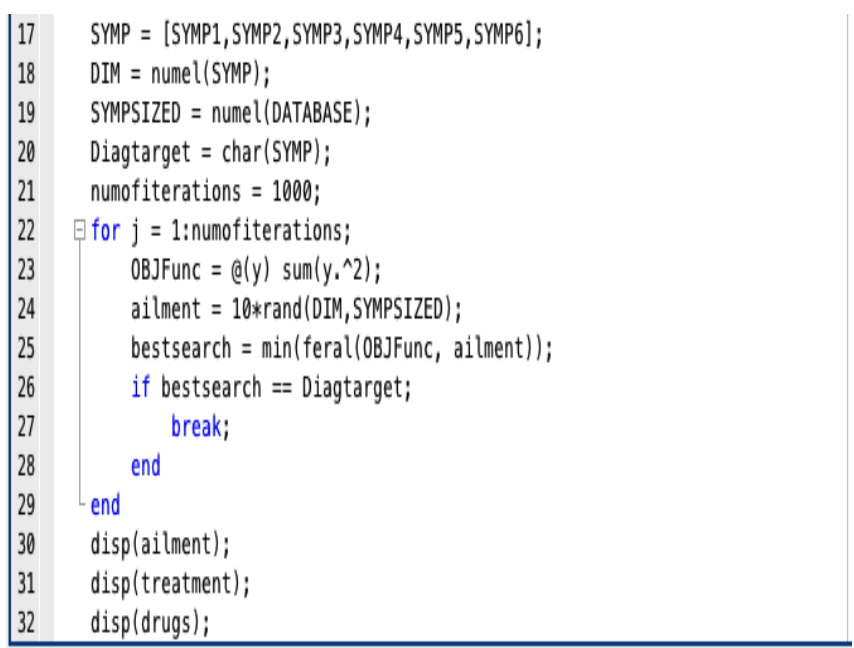

Fig. 7: Pure random search algorithm to identify an ailment with a given symptom

The number of symptoms selected for a patient is saved in "SYNP", which stores selected number of symptoms identified by the system as the valid number of symptoms to fetch an ailment. The control uses a random search equivalent to methods of text mining using Matlab programming language (Figure 6 and 7) 


$$
g(y): g(y)=\sum y(i)^{100 \backslash 50} \ldots
$$

The search finds the best match for the ailment relating to the symptoms and produces or suggest the right treatment and drugs. The dimension of the search procedure depends on the symptoms selected. Random number of symptoms are generated to meet the target solution to an ailment matching the once in the system. The objective function equation is defined and corresponding ailments are generated randomly. The best search that relates to the given diagnosis target is then selected, if it satisfies the target ailment and the loop is broken. The right ailment is displayed together with the corresponding treatments and drugs.

\section{Conclusion}

The paper sort to develop a computer aided medical diagnosis for the treatment of sexually transmitted diseases such as gonorrhea. The aim and objectives of the project have been met laterality and discussed in most section of the paper. Based on the output of the interphases speed in execution, it's obvious that there would be a great relief for the doctors and even non-experts to diagnose some STDs and prescribe drugs for such diseases. Above all, computer can be the most effective and faster way in the diagnosis and treatment of sexually transmitted diseases. Despite the above, a conclusion can still be drawn by saying that STDs (gonorrhea) rate can only be reduced by avoiding sexual promiscuity, rapidly eradicating the bacteria and spirochete from infected individuals by means of early diagnosis and treatment, creating awareness through sex education. Prevention they say is better than cure, so the use of mechanical prophylaxis (condoms) and chemoprophylaxis (e.g. applying penicillin after exposure) provides only partially protection but total abstinence provides total protection. STAY HEALTHY TO BE WEALTHY. It is highly recommended that this program Computer Aided Medical Diagnosis for the Treatment of Sexually Transmitted Diseases be used in all the clinics, and most especially in an area where there is shortage of medical personnel.

\section{Acknowledgment}

The authors would like to thank the Unity Hospital in Keffi, Nassarwa state of Nigeria for taking the time to communicate the problems they are facing and making this project possible.

\section{References}

[1] Rella Adams and Pam Duchene. Computerization of patient acuity and nursing care planning: New approach to improved patient care and costeffective staffing. Journal of Nursing Administration, 15(4):11-17, 1985.

[2] Word Bank. Review of banks activities in the health sector in europe and central asia. 1990.

[3] Joaquin A Blaya, Hamish SF Fraser, and Brian Holt. E-health technologies show promise in developing countries. Health Affairs, 29(2):244-251, 2010.

[4] David Dean, Kyoung-June Min, and Angus Bond. Computer aided design of large-format prefabricated cranial plates. Journal of Craniofacial Surgery, 14(6):819-832, 2003.

[5] Robert Feyen, Yili Liu, Don Chaffin, Glenn Jimmerson, and Brad Joseph. Computer-aided ergonomics: a case study of incorporating ergonomics analyses into workplace design. Applied ergonomics, 31(3):291-300, 2000.
[6] Richard Harvey, S. Y Salih, and Alan E Read. Organic and functional disorders in 2000 gastroenterology outpatients. The Lancet, 321(8325):632634, 1983.

[7] R Hillyard and Ie Braid. Analysis of dimensions and tolerances in computeraided mechanical design. Computer Aided Design, 10(3):161-166, 1978.

[8] William L Jorgensen, Juliana Ruiz-Caro, Julian Tirado-Rives, Aravind Basavapathruni, Karen S Anderson, and Andrew D Hamilton. Computeraided design of non-nucleoside inhibitors of hiv-1 reverse transcriptase. Bioorganic \& medicinal chemistry letters, 16(3):663-667, 2006.

[9] George F Luger and William A. Stubblefield. Artificial intelligence and the design of expert systems, benjemmin, 1989.

[10] Michael Moran. Three faces of the health care state. Journal of Health Politics, Policy and Law, 20(3):767-781, 1995.

[11] Cliff Moughtin, Paola Signoretta, and Kate McMahon Moughtin. Urban design: health and the therapeutic environment. Routledge, 2009.

[12] Nelishia Pillay. A generative hyper-heuristic for deriving heuristics for classical artificial intelligence problems. In Advances in Nature and Biologically Inspired Computing, pages 337-346. Springer, 2016.

[13] Jose Reina, Emmanuel Lacroix, Scott D Hobson, Gregorio FernandezBallester, Vladimir Rybin, Markus S Schwab, Luis Serrano, and Cayetano Gonzalez. Computer-aided design of a pdz domain to recognize new target sequences. Nature Structural \& Molecular Biology, 9(8):621-627, 2002.

[14] Anne Verroust, Franc, ois Schonek, and Dieter Roller.Rule-oriented method for parameterized computer-aided design. Computer-Aided Design, 24(10):531-540, 1992. 